\title{
Antibiotikaresistenzen: Eine ständig zunehmende Gefahr für Mensch und Tier und mögliche Auswege aus dem sich abzeichnenden Dilemma
}

\author{
Jürgen Wallmann • Thomas Heberer
}

Published online: 18 January 2014

(C) Bundesamt für Verbraucherschutz und Lebensmittelsicherheit (BVL) 2014

Antibiotika sind natürliche oder synthetische Moleküle, die Bakterien abtöten oder das Bakterienwachstum hemmen. Sie haben unseren Umgang mit Infektionskrankheiten des Menschen seit nun mehr als 80 Jahren stark beeinflusst. Darüber hinaus werden sie auch in der Agrar- und Fischwirtschaft intensiv verwendet. Der z. T. bis heute intensive und auch kritiklose Einsatz von Antibiotika in allen Bereichen hat dazu geführt, dass bereits heute Experten von einer „post-antibiotischen Ära“ sprechen.

Antibiotika haben die Infektionstherapie revolutioniert, indem es möglich wurde, Krankheiten zu heilen, die bis dato fast immer zum Tode führten. Da die Wirksamkeit der Antibiotika zunächst in keiner Weise in Frage zu stellen war, erfolgte teilweise der bis heute andauernde ungehemmte Einsatz. Schon bald wurde aber deutlich, dass Antibiotika sich „,verbrauchen“, indem sie ihre Wirkung gegen Bakterien verlieren, weil diese Unempfindlichkeitsmechanismen (Resistenz) gegen Antibiotika entwickeln. Es folgte eine Zeitspanne von etwa 60 Jahren, in denen fortlaufend neue Moleküle gefunden wurden, die es ermöglichten, resistent gewordene Bakterien weiterhin abzutöten oder in ihrem Wachstum zu hemmen. Insbesondere seit Mitte der sechziger und Anfang der neunziger Jahre scheint sich durch den zunehmenden Einsatz von Antibiotika in der Human- und Veterinärmedizin weltweit ein

Dr. J. Wallmann (凶) · Prof. Dr. T. Heberer Bundesamt für Verbraucherschutz und Lebensmittelsicherheit, Mauerstr. 39-42, 10117 Berlin, Germany

e-mail: juergen.wallmann@bvl.bund.de
Trend wachsender Resistenzentwicklung, eine Zunahme des Auftretens von Resistenzen und der Resistenzausbreitung bei bakteriellen Infektionserregern, abzuzeichnen. Der bereits zu Beginn sowie am Ende der achtziger Jahre von Wissenschaftlern befürchtete Therapienotstand bei bakteriellen Infektionen - weitestgehend aber als marginal klassifiziert - wurde durch die Einführung der neuen Cephalosporine der 3. Generation und der Fluorchinolone sowie der Carbapeneme aus der damaligen Diskussion verdrängt. Die Entwicklung neuer Wirkstoffgruppen ist aktuell jedoch nicht mehr erkennbar.

Der wirtschaftliche Nutzen dieser Substanzen für die Volkswirtschaften bleibt unbestritten. Dennoch muss die Frage gestellt werden, ob diese wertvollen Substanzen heute nicht zu einem viel zu günstigen Preis zu erwerben sind. Die Möglichkeit, eine wirkstoffgleiche Kopie eines bereits unter einem Markennamen auf dem Markt befindlichen Medikaments zu verkaufen, hat z. T. zu einem Preisverfall bei Antibiotika geführt. Somit sind Antibiotika heute häufig billiger als Hygienemaßnahmen oder die Implementierung adäquater Haltungsformen bei Tieren.

Betroffen von dieser negativen Entwicklung sind dabei aber nicht nur die für Mensch und Tier pathogenen bakteriellen Infektionserreger, sondern entsprechend auch apathogene, ubiquitär vorkommende Bakterienspezies, die möglicherweise einen „,pool“ genetisch determinierter Resistenzen aufweisen und diese Resistenzgene durch horizontalen Resistenzgentransfer an pathogene Bakterienarten weitergeben.

Das Risiko der Konfrontation des Menschen mit resistenten Bakterien von landwirtschaftlichen 
Nutztieren durch Selektion resistenter Keime bei therapierten Tieren kann sich einerseits auf dem Wege kontaminierter Lebensmittel tierischer Herkunft und durch Resistenzselektion in der humanen Darmflora durch antimikrobielle Rückstände ergeben. Andererseits ist die Übertragung resistenter Keime bei behandelten Nutz- und Heimtieren auf den Menschen durch direkten Tierkontakt, Austausch von Resistenzgenen mit humanpathogenen Keimen sowie Kolonisation mit resistenten Keimen und Ausscheidung resistenter Keime aus dem Darm der Tiere möglich. Erwähnt sei an dieser Stelle zudem, dass resistente Keime durch engen Kontakt der Tierhalter und Tierärzte mit Nutz- und vor allem Heimtieren auch vom Menschen auf Tiere übertragen und somit weiter verbreitet werden. Was den Menschen betrifft, so wird durch die ständige Aufnahme von Bakterien mit möglicherweise übertragbarer Resistenz aus dem Bereich der Tierproduktion die Wahrscheinlichkeit für die weitere Übertragung von Resistenzeigenschaften erhöht. Die Globalisierung durch z. B. stetig zunehmende Fernreisen oder länderübergreifende Geschäftsverbindungen führen auch zu einer Globalisierung des bakteriellen Ökosystems, mit weitreichenden Konsequenzen wie z. B. umfangreichen Interaktionen zwischen ambulanter Praxis und Hospitaleinrichtungen sowie zwischen Mensch und Tier.

Damit die Verknüpfung von Antibiotikaanwendung und Resistenzentstehung verstanden werden kann, ist es unabdingbar, sowohl quantitative Daten zur Resistenz der verschiedenen Bakterienspezies bei den unterschiedlichen Tierarten wie auch spezifizierte Daten zu den Verbrauchsmengen an Antibiotika zur Verfügung zu haben.

Zur Vermeidung des unkritischen Einsatzes von Antibiotika ohne mikrobiologische Absicherung sowie zur Durchführung einer effizienten antimikrobiellen Therapie und Minimierung der Gefahr der Resistenzselektion erscheint es nach wie vor wichtig, den aktuell konkreten Stellenwert der einzelnen Antibiotika (Antibiotikagruppen) in einem Konsens von Fachgremien, Arzneimittelherstellern und $\mathrm{Zu}-$ lassungsbehörden bei den verschiedenen bakteriellen Infektionskrankheiten klar zu definieren. Diese durch formale Prozesse zu entwickelnden standardisierten Spezifikationen für die Therapie und die Kontrolle von bakteriellen Infektionskrankheiten bieten die beste wissenschaftliche Grundlage für die Bekämpfung der Resistenzentwicklung und -ausbreitung.

Die Implementierung von Richtlinien, z. B. auch zur Bewertung bedeutender Antibiotikaindika-tionen, sowie valide Behandlungsstrategien können einen Beitrag zur Optimierung des Antibiotikaein- satzes leisten. Grundsätzlich kann aber nur der restriktive und verantwortungsvolle Einsatz der vorhandenen antimikrobiell wirksamen Substanzen einem „Resistenzkollaps“ wirkungsvoll entgegenwirken. Jede überflüssige und ungezielte oder nicht zu Ende geführte Antibiotikatherapie begünstigt z. B. eine Resistenzselektion und kann den Gen-Pool mit Antibiotikaresistenzen bei Infektionserregern vergrößern.

Im landwirtschaftlichen Bereich muss der Ansatz zur Reduktion der Resistenzentwicklung jedoch umfassender ,gedacht“ werden. Sind die heutigen Haltungs- und Managementbedingungen noch zeitgemäß, und inwieweit tragen sie zu einer Resistenzentwicklung und -ausbreitung bei? Müssen die Zuchtziele in der Landwirtschaft nicht überdacht bzw. verändert werden, und wie sieht es mit der Preisgestaltung bei Antibiotika aus? Diese Grundsatzfragen müssen gestellt und auch beantwortet werden. Genauso ist auf der Seite der Verbraucher weitere Aufklärung und die zwingende Einhaltung von adäquaten Verhaltensregeln in der Küche bei der Zubereitung von und im Umgang mit Speisen im Sinne der guten Küchenhygiene notwendig.

Die derzeit zur Verwendung bei Mensch und Tier geeigneten Antibiotika ermöglichen aktuell noch weitestgehend die Kontrolle der meisten bakteriellen Infektionen. Wenn wir aber keine grundlegenden Änderungen herbeiführen, stellt sich konkret schon jetzt die Frage: „Wie lange noch?“.

Die Beziehung zwischen den Parametern „Anwendung“ und „Resistenzentwicklung“ ist ausgesprochen komplex, so dass der alleinige reduzierte Antibiotikaeinsatz nicht in jedem Fall zwangsläufig zu einer Reduktion von Resistenzprävalenzen führen muss. Deshalb sind plakative Forderungen, die nur die Reduktion berücksichtigen, nicht wirklich zielführend.

Das Bundesamt für Verbraucherschutz und Lebensmittelsicherheit hat im Rahmen seines alljährlich veranstalteten Symposiums diese Thematik aufgegriffen und mit nationalen und internationalen Experten umfänglich erläutert und diskutiert. Das Thema „Antibiotikaresistenz im Spannungsfeld zwischen Humanmedizin, Tiermedizin und Verbraucherschutz" stand im Mittelpunkt des ersten Tages des am 5. und 6. November 2013 in Berlin veranstalteten Symposiums mit dem Titel „Herausforderungen 2014: Tierarzneimittel im Fokus - Nutzen, Risiken, Resistenzen“" Ergebnisse dieses Symposiums sind in dem Beitrag „Antibakterielle Resistenzen in Deutschland: Lage, Ursachen und Gegenmaßnahmen“ in diesem Heft zusammengefasst. 